SESSION F

ASTRONOMICAL REFRACTION PROBLEMS 


\title{
THE PRESENT STATE AND FUTURE OF THE ASTRONOMICAL REFRACTION INVESTIGATIONS*
}

\author{
G. TELEK I \\ Astronomical Observatory, Belgrade, Yugoslavia
}

\begin{abstract}
Astronomical refraction is one of the oldest astronomical problems, which has been investigated many times, but the existing knowledge of refraction is not sufficient to meet modern requirements for accurate astrometric data. The present investigations cast light on several facts which might help the advancement in the calculation of astronomical refraction.

(2) There are several questions concerning the calculation of the astronomical refraction, from the laws of refraction to meteorological factors. These difficulties have prevented the development of a satisfactory refraction theory for a long time, leading to the conservation of old methods, but the accuracy of the refraction determinations has not increased. We are using old refraction theories and tables based on them for the most part, while the anomalous refraction is not considered. Seeing that the calculated refraction values are not acceptable, we are determining the formal refraction corrections directly from astrometric measurements, but these corrections are not real from the physical point of view.

The present uncertainty in the calculated refraction is of the order of 0.01 , increasing with zenith distance. For observations near the horizon the existing refraction theories cannot give acceptably accurate values.

(3) The greatest progress in the knowledge of refraction influences would be achieved if instead of mean meteorological data we could use direct measurement. There are some propositions of this kind.

The new refraction tables are in preparation. We are very careful in taking into consideration the local characteristics and the correct calculation of the chromatic refraction.

Frankly speaking, we must say that it is practically impossible to calculate a real influence of refraction and for this reason certain precautions are advisable. A careful positioning of the instrument may simplify the density field and thus eliminate the greatest part of the anomalous refraction. But the best proposition is to observe at small zenith distances only, not larger than $45^{\circ}$ if possible.

(4) Our conclusion is that, in current astrometric practice, we must continue refraction investigations and in addition to the refraction at optical wavelengths, it is absolutely necessary to investigate the radio and laser-refraction too.
\end{abstract}

* The full text of this paper will appear in Publications de l'Observatoire Astronomique de Béograd, No. 18.

Gliese, Murray, and Tucker. 'New Problems in Astrometry', 235-236. All Rights Reserved.

Copyright $\mathrm{C} 1974$ by the IAU. 


\section{DISCUSSION}

Van Herk: The geodesists suffer from difficulties with refraction as well as the astronomers do. They have formed a study commission with, as co-ordinator, Dr E. Tengström, Geodetic Institute, Uppsala University, Hällby, Uppsala 75590, Sweden.

Maybe the two study commissions could aid each other.

Teleki: I will propose the co-operation.

Strand: Some 20 years ago refraction at large distances was measured at the Dearborn Observatory from separation of craters on the moon, as the moon was rising over Lake Michigan. Comparison with the theoretical refraction and using available atmospheric data, showed clearly that the actual refraction could differ as much as $5 \%$ from the computed value.

Teleki: It can be seen that there are many problems connected with the refraction.

Kovalevsky: It is to be remarked that theoretical and observational material analysed by Miss Hopfield showed that the dry component of refraction in distance (for laser measurements) can be modelled from observations of temperature and pressure at the site of the observer to an accuracy of about $1 \mathrm{~cm}$. 